\title{
Current treatments and scientific advancements to combat the epidemic novel coronavirus.
}

\author{
Francesco Ferrara ${ }^{1}$, Chiara Pelliccia ${ }^{2}$, and Antonio Vitiello ${ }^{3}$ \\ ${ }^{1}$ USL 1 Umbria \\ ${ }^{2}$ Azienda Unità Sanitaria Locale Umbria 2 \\ ${ }^{3}$ Azienda Unità Sanitaria Locale Umbria 1
}

April 28, 2020

\begin{abstract}
An acute respiratory disease, caused by a novel coronavirus (SARS-CoV-2) has spread throughout China and other countries and received worldwide attention. On 30 January 2020, World Health Organization (WHO) officially declared the SARS-CoV-2 epidemic as a public health emergency of international concern. At the moment of writing this article (April 2020) a total of 2.240.191 cases confirmed worldwide since the outbreak and 153.822 deaths in 166 countries or regions confirmed cases globally had been reported. Meanwhile, several independent research groups have identified that SARS-CoV-2 has highly identical genome with SARS-COV-1. The novel coronavirus uses the same receptor, angiotensin-converting enzyme 2 (ACE2) as that for SARS-CoV, and mainly spreads through the respiratory tract. The clinical symptoms of patients include fever, cough, fatigue and a small population of patients appeared gastrointestinal infection symptoms. Currently, there are few specific therapeutic strategies, but several potent candidates of antivirals and repurposed drugs are under urgent investigation. In this review, we summarized the current treatment and scientific advancements to combat the epidemic novel coronavirus. Keywords: SARS-CoV-2, treatment, coronavirus, infection, pneumonia
\end{abstract}

\section{Hosted file}

Current treatments and scientific advancements to combat the epidemic novel coronavirus..pdf available at https : //authorea.com/users/310209/articles/444911-current-treatments-and-scientificadvancements-to-combat-the-epidemic-novel-coronavirus 